\title{
A generalization of Banach's contraction principle for nonlinear contraction in a partial metric space
}

\author{
Wasfi Shatanawi ${ }^{a}$, Hemant Kumar Nashine ${ }^{b, *}$ \\ ${ }^{a}$ Department of Mathematics, Hashemite University, Zarqa, Jordan. \\ ${ }^{b}$ Department of Mathematics, Disha Institute of Management and Technology, Satya Vihar, Vidhansabha-Chandrakhuri Marg, \\ Naradha, Mandir Hasaud, Raipur-492101 (Chhattisgarh), India.
}

\author{
This paper is dedicated to Professor Lj. B. Ćirić \\ Communicated by Professor V. Berinde
}

\begin{abstract}
We establish a fixed point theorem for nonlinear contraction in a complete partial metric space. Our result generalizes the Banach type fixed point theorem in a partial metric space in the sense of Matthews.(C)2012 NGA. All rights reserved.
\end{abstract}

Keywords: Partial metric space, Banach principle, Fixed Point Theory.

2010 MSC: Primary 54H25; Secondary 47H10.

\section{Introduction and Preliminaries}

In 1994, Matthews [22] introduced the notion of a partial metric space in such a way that each object doesn't necessarily have to have a zero distance from itself. Also, Matthews [22] studied the Banach's contraction principle in such space. After then, many authors studied many fixed point results in partial metric spaces ( see $[1-5,17,19-25,27]$ ).

In this section, we give the necessarily definitions and lemmas for the partial metric spaces.

Definition 1.1. 22] A partial metric on a nonempty set $X$ is a function $p: X \times X \rightarrow \mathbb{R}^{+}$such that for all $x, y, z \in X:$

$\left(\mathrm{p}_{1}\right) x=y \Longleftrightarrow p(x, x)=p(x, y)=p(y, y)$,

\footnotetext{
${ }^{*}$ Corresponding author

Email addresses: swasfi@maktoob.com (Wasfi Shatanawi), drhknashine@gmail.com (Hemant Kumar Nashine)
} 
$\left(\mathrm{p}_{2}\right) p(x, x) \leq p(x, y)$,

$\left(\mathrm{p}_{3}\right) p(x, y)=p(y, x)$,

$\left(\mathrm{p}_{4}\right) p(x, y) \leq p(x, z)+p(z, y)-p(z, z)$.

A partial metric space is a pair $(X, p)$ such that $X$ is a nonempty set and $p$ is a partial metric on $X$.

Each partial metric $p$ on $X$ generates a $T_{0}$ topology $\tau_{p}$ on $X$. The set $\left\{B_{p}(x, \varepsilon): x \in X, \varepsilon>0\right\}$, where $B_{p}(x, \varepsilon)=\{y \in X: p(x, y)<p(x, x)+\varepsilon\}$ for all $x \in X$ and $\varepsilon>0$ forms the base of $\tau_{p}$

If $p$ is a partial metric on $X$, then the function $p^{s}: X \times X \rightarrow \mathbb{R}^{+}$given by

$$
p^{s}(x, y)=2 p(x, y)-p(x, x)-p(y, y)
$$

is a metric on $X$.

Definition 1.2. [22] Let $(X, p)$ be a partial metric space. Then:

1. A sequence $\left\{x_{n}\right\}$ in a partial metric space $(X, p)$ converges to a point $x \in X$ if and only if $p(x, x)=$ $\lim _{n \rightarrow \infty} p\left(x, x_{n}\right)$.

2. A sequence $\left\{x_{n}\right\}$ in a partial metric space $(X, p)$ is called a Cauchy sequence if there exists (and is finite) $\lim _{n, m \rightarrow \infty} p\left(x_{n}, x_{m}\right)$.

3. A partial metric space $(X, p)$ is said to be complete if every Cauchy sequence $\left\{x_{n}\right\}$ in $X$ converges, with respect to $\tau_{p}$, to a point $x \in X$ such that $p(x, x)=\lim _{n, m \rightarrow \infty} p\left(x_{n}, x_{m}\right)$

The following lemma plays a major role in proving our main results.

Lemma 1.3. [22] Let $(X, p)$ be a partial metric space.

1. $\left\{x_{n}\right\}$ is a Cauchy sequence in $(X, p)$ if and only if it is a Cauchy sequence in the metric space $\left(X, p^{s}\right)$.

2. A partial metric space $(X, p)$ is complete if and only if the metric space $\left(X, p^{s}\right)$ is complete. Furthermore, $\lim _{n \rightarrow \infty} p^{s}\left(x_{n}, x\right)=0$ if and only if

$$
p(x, x)=\lim _{n \rightarrow \infty} p\left(x_{n}, x\right)=\lim _{n, m \rightarrow \infty} p\left(x_{n}, x_{m}\right) .
$$

Lemma 1.4. [20] Let $x_{n} \rightarrow z$ as $n \rightarrow+\infty$ in a partial metric space $(X, p)$ where $p(z, z)=0$, then $\lim _{n \rightarrow+\infty} p\left(x_{n}, y\right)=p(z, y)$ for every $y \in X$.

Ćirić is one of the pioneer workers in the field of fixed point theory. Ćirić established and studied many fixed point theorems for mappings satisfying different contractive conditions in complete metric spaces, for example see [8]-[16]. Then after, many authors studied many fixed point theorems by using the different types of Ćirić contractions, for example see [6, 17, 27].

In this paper, we establish some fixed point results for strong Ćirić type quasi contractions in the setting of a complete partial metric space. Also, we introduce an example to support the useability of our results.

\section{The Main Result}

We start our work by giving a fixed point theorem for nonlinear contraction in a partial metric space.

Theorem 2.1. Let $(X, p)$ be a complete partial metric space and $T: X \rightarrow X$ be a mapping satisfying

$$
\begin{aligned}
p(T x, T y) \leq & \max \left\{p(x, y), p(x, T x), p(y, T y), \frac{1}{2}[p(x, T y)+p(T x, y)]\right\} \\
& -\psi(p(x, y), p(x, T x)), \quad \forall x, y \in X,
\end{aligned}
$$

where $\psi:[0, \infty) \times[0,+\infty) \rightarrow[0, \infty)$ is a continuous mapping such that $\psi(t, s)=0$ if and only if $t=s=0$. Then $T$ has a unique fixed point. 
Proof. Let $x_{0}$ be an arbitrary point in $X$. We choose $x_{1} \in X$ such that $x_{1}=T x_{0}$. By continuing in the same way, we construct a sequence $\left(x_{n}\right)$ in $X$ such that

$$
x_{n+1}=T x_{n}, \quad n=0,1,2,3, \cdots .
$$

If there exists $n \in \mathbf{N}$ such that $p\left(x_{n}, x_{n+1}\right)=0$, then by $\left(p_{1}\right)$ and $\left(p_{2}\right)$ we have $x_{n}=x_{n+1}=T x_{n}$. Hence $x_{n}$ is a fixed point of $T$. Now, we assume that $p\left(x_{n}, x_{n+1}\right) \neq 0$ for all $n \geq 0$. Thus, by (2.1), we have

$$
\begin{aligned}
& p\left(x_{n+1}, x_{n+2}\right) \\
= & p\left(T x_{n}, T x_{n+1}\right) \\
\leq & \max \left\{p\left(x_{n}, x_{n+1}\right), p\left(x_{n}, T x_{n}\right), p\left(x_{n+1}, T x_{n+1}\right), \frac{1}{2}\left[p\left(x_{n}, T x_{n+1}\right)+p\left(T x_{n}, x_{n+1}\right)\right]\right\} \\
& -\psi\left(p\left(x_{n}, x_{n+1}\right), p\left(x_{n}, T x_{n}\right)\right) \\
= & \max \left\{p\left(x_{n}, x_{n+1}\right), p\left(x_{n+1}, x_{n+2}\right), \frac{1}{2}\left[p\left(x_{n}, x_{n+2}\right)+p\left(x_{n+1}, x_{n+1}\right)\right]\right\} \\
& -\psi\left(p\left(x_{n}, x_{n+1}\right), p\left(x_{n}, x_{n+1}\right)\right) .
\end{aligned}
$$

By $\left(p_{4}\right)$, we have

$$
p\left(x_{n}, x_{n+2}\right)+p\left(x_{n+1}, x_{n+1}\right) \leq p\left(x_{n}, x_{n+1}\right)+p\left(x_{n+1}, x_{n+2}\right) .
$$

Therefore,

$$
\begin{aligned}
& \max \left\{p\left(x_{n}, x_{n+1}\right), p\left(x_{n+1}, x_{n+2}\right), \frac{1}{2}\left[p\left(x_{n}, x_{n+2}\right)+p\left(x_{n+1}, x_{n+1}\right)\right]\right\} \\
\leq & \max \left\{p\left(x_{n}, x_{n+1}\right), p\left(x_{n+1}, x_{n+2}\right)\right\} .
\end{aligned}
$$

By (2.2) and (2.3), we have

$$
p\left(x_{n+1}, x_{n+2}\right) \leq \max \left\{p\left(x_{n}, x_{n+1}\right), p\left(x_{n+1}, x_{n+2}\right)\right\}-\psi\left(p\left(x_{n}, x_{n+1}\right), p\left(x_{n}, x_{n+1}\right)\right) .
$$

If $\max \left\{p\left(x_{n}, x_{n+1}\right), p\left(x_{n+1}, x_{n+2}\right)\right\}=p\left(x_{n+1}, x_{n+2}\right)$, then from 2.15), we have

$$
p\left(x_{n+1}, x_{n+2}\right) \leq p\left(x_{n+1}, x_{n+2}\right)-\psi\left(p\left(x_{n}, x_{n+1}\right), p\left(x_{n}, x_{n+1}\right)\right)<p\left(x_{n+1}, x_{n+2}\right) .
$$

which is a contradiction since $\psi\left(p\left(x_{n}, x_{n+1}\right), p\left(x_{n}, x_{n+1}\right)\right)=0$ and so $p\left(x_{n}, x_{n+1}\right)=0$, that $x_{n}=x_{n+1}$. Therefore, we have $\max \left\{p\left(x_{n}, x_{n+1}\right), p\left(x_{n+1}, x_{n+2}\right)\right\}=p\left(x_{n}, x_{n+1}\right)$ and hence

$$
p\left(x_{n+1}, x_{n+2}\right) \leq p\left(x_{n}, x_{n+1}\right)-\psi\left(p\left(x_{n}, x_{n+1}\right), p\left(x_{n}, x_{n+1}\right)\right) \leq p\left(x_{n}, x_{n+1}\right) .
$$

By (2.6), we have $\left\{p\left(x_{n}, x_{n+1}\right)\right\}$ is a non-increasing sequence of positive real numbers. Thus, there exists $r \geq 0$ such that

$$
\lim _{n \rightarrow \infty} p\left(x_{n}, x_{n+1}\right)=r .
$$

Letting $n \rightarrow \infty$ in 2.6) and using (2.7) and the properties of $\psi$, we have $r \leq r-\psi(r, r)$. Thus $\psi(r, r)=0$ and hence $r=0$. Therefore

$$
\lim _{n \rightarrow \infty} p\left(x_{n}, x_{n+1}\right)=0 .
$$

Our next step is to prove that

$$
\lim _{n, m \rightarrow \infty} p\left(x_{n}, x_{m}\right)=0 .
$$

Suppose the contrary, that is,

$$
\lim _{n, m \rightarrow \infty} p\left(x_{n}, x_{m}\right) \neq 0 .
$$


Then there exists $\epsilon>0$ for which we can find two subsequences $\left\{x_{m(k)}\right\},\left\{x_{n(k)}\right\}$ of $\left\{x_{n}\right\}$ such that $n(k)$ is the smallest index for which

$$
n(k)>m(k)>k, p\left(x_{n(k)}, x_{m(k)}\right) \geq \epsilon .
$$

This means that

$$
p\left(x_{n(k)-1}, x_{m(k)}\right)<\epsilon .
$$

From 2.9 and 2.10$)$, we have

$$
\begin{aligned}
\epsilon \leq p\left(x_{n(k)}, x_{m(k)}\right) & \leq p\left(x_{n(k)}, x_{n(k)-1}\right)+p\left(x_{n(k)-1}, x_{m(k)}\right)-p\left(x_{n(k)-1}, x_{n(k)-1}\right) \\
& \leq p\left(x_{n(k)}, x_{n(k)-1}\right)+p\left(x_{n(k)-1}, x_{m(k)}\right) \\
& <\epsilon+p\left(x_{n(k)}, x_{n(k)-1}\right)
\end{aligned}
$$

Taking $k \rightarrow \infty$ and using (2.8), we get

$$
\lim _{k \rightarrow \infty} p\left(x_{n(k)}, x_{m(k)}\right)=\epsilon
$$

By $\left(p_{3}\right)$ and $\left(p_{4}\right)$, we have

$$
\begin{aligned}
& p\left(x_{n(k)}, x_{m(k)}\right) \\
\leq & p\left(x_{n(k)}, x_{n(k)+1}\right)+p\left(x_{n(k)+1}, x_{m(k)}\right)-p\left(x_{n(k)+1}, x_{n(k)+1}\right) \\
\leq & p\left(x_{n(k)}, x_{n(k)+1}\right)+p\left(x_{n(k)+1}, x_{m(k)}\right) \\
\leq & p\left(x_{n(k)}, x_{n(k)+1}\right)+p\left(x_{n(k)+1}, x_{m(k)+1}\right)+p\left(x_{m(k)+1}, x_{m(k)}\right)-p\left(x_{m(k)+1}, x_{m(k)+1}\right) \\
\leq & p\left(x_{n(k)}, x_{n(k)+1}\right)+p\left(x_{n(k)+1}, x_{m(k)+1}\right)+p\left(x_{m(k)+1}, x_{m(k)}\right) \\
\leq & 2 p\left(x_{n(k)}, x_{n(k)+1}\right)+p\left(x_{n(k)}, x_{m(k)+1}\right)+p\left(x_{m(k)+1}, x_{m(k)}\right)-p\left(x_{n(k)}, x_{n(k)}\right) \\
\leq & 2 p\left(x_{n(k)}, x_{n(k)+1}\right)+p\left(x_{n(k)}, x_{m(k)+1}\right)+p\left(x_{m(k)+1}, x_{m(k)}\right) \\
\leq & 2 p\left(x_{n(k)}, x_{n(k)+1}\right)+p\left(x_{n(k)}, x_{m(k)}\right)+2 p\left(x_{m(k)+1}, x_{m(k)}\right)-p\left(x_{m(k)}, x_{m(k)}\right) \\
\leq & 2 p\left(x_{n(k)}, x_{n(k)+1}\right)+p\left(x_{n(k)}, x_{m(k)}\right)+2 p\left(x_{m(k)+1}, x_{m(k)}\right)
\end{aligned}
$$

Taking $k \rightarrow \infty$ in the above inequalities and using (2.8), (2.11), we get that

$$
\begin{aligned}
& \lim _{k \rightarrow \infty} p\left(x_{n(k)}, x_{m(k)}\right)=\lim _{k \rightarrow \infty} p\left(x_{n(k)+1}, x_{m(k)}\right) \\
= & \lim _{k \rightarrow \infty} p\left(x_{n(k)}, x_{m(k)+1}\right)=\lim _{k \rightarrow \infty} p\left(x_{n(k)+1}, x_{m(k)+1}\right)=\epsilon
\end{aligned}
$$

Now, from 2.1), we have

$$
\begin{aligned}
& p\left(x_{m(k)+1}, x_{n(k)+1}\right)=p\left(T x_{m(k)}, T x_{n(k)}\right) \\
\leq & \max \left\{p\left(x_{m(k)}, x_{n(k)}\right), p\left(x_{m(k)}, T x_{m(k)}\right), p\left(x_{n(k)}, T x_{n(k)}\right),\right. \\
& \left.\frac{1}{2}\left(p\left(x_{m(k)}, T x_{n(k)}\right)+p\left(T x_{m(k)}, x_{n(k)}\right)\right)\right\}-\psi\left(p\left(x_{m(k)}, x_{n(k)}\right), p\left(x_{m(k)}, T x_{m(k)}\right)\right) \\
= & \max \left\{p\left(x_{m(k)}, x_{n(k)}\right), p\left(x_{m(k)}, x_{m(k)+1}\right), p\left(x_{n(k)}, x_{n(k)+1}\right),\right. \\
& \left.\frac{1}{2}\left(p\left(x_{m(k)}, x_{n(k)+1}\right)+p\left(x_{m(k)+1}, x_{n(k)}\right)\right)\right\}-\psi\left(p\left(x_{m(k)}, x_{n(k)}\right), p\left(x_{m(k)}, x_{m(k)+1}\right)\right)
\end{aligned}
$$

On letting $k \rightarrow \infty$ in 2.13 and using $2.8,2.12$ and the properties of $\psi$, we have

$$
\epsilon \leq \epsilon-\psi(\epsilon, \epsilon)<\epsilon
$$

which is a contradiction. So, we have

$$
\lim _{n, m \rightarrow \infty} p\left(x_{n}, x_{m}\right)=0 .
$$

Since $\lim _{n, m \rightarrow \infty} p\left(x_{n}, x_{m}\right)$ exists and finite, we conclude that $\left(x_{n}\right)$ is a Cauchy sequence in $(X, p)$. 
By (1.1), we have $p^{s}\left(x_{n}, x_{m}\right) \leq 2 p\left(x_{n}, x_{m}\right)$. Therefore

$$
\lim _{n, m \rightarrow \infty} p^{s}\left(x_{n}, x_{m}\right)=0 .
$$

Thus, by Lemma 1.3, $\left\{x_{n}\right\}$ is a Cauchy sequence in both $\left(X, p^{s}\right)$ and $(X, p)$. Thus, there exists $x \in X$ such that $\lim _{n \rightarrow \infty} p^{s}\left(x_{n}, x\right)=0$ if and only if

$$
p(x, x)=\lim _{n \rightarrow \infty} p\left(x_{n}, x\right)=\lim _{n, m \rightarrow \infty} p\left(x_{n}, x_{m}\right)=0 .
$$

Now, we prove that $x$ is a fixed point of $T$. From (2.1), we have

$$
\begin{aligned}
& p\left(T x, x_{n+1}\right)=p\left(T x, T x_{n}\right) \\
\leq & \max \left\{p\left(x, x_{n}\right), p(x, T x), p\left(x_{n}, T x_{n}\right), \frac{1}{2}\left(p\left(T x, x_{n}\right)+p\left(x, T x_{n}\right)\right\}-\psi\left(p\left(x, x_{n}\right), p(x, T x)\right)\right. \\
= & \max \left\{p\left(x, x_{n}\right), p(x, T x), p\left(x_{n}, x_{n+1}\right), \frac{1}{2}\left(p\left(T x, x_{n}\right)+p\left(x, x_{n+1}\right)\right\}-\psi\left(p\left(x, x_{n}\right), p(x, T x)\right) .\right.
\end{aligned}
$$

Letting $n \rightarrow \infty$ in the above inequality, and using Lemma (1.4) we obtain

$$
p(x, T x) \leq p(x, T x)-\psi(0, p(x, T x)) .
$$

Hence $\psi(0, p(x, T x))=0$. Thus $p(x, T x)=0$. By $\left(p_{1}\right)$ and $\left(p_{2}\right)$, we have $T x=x$. Therefore $x$ is a fixed point of $T$. To prove the uniqueness of the fixed point. Suppose that $y$ is another fixed point of $T$. From (2.1), we have

$$
p(x, y)=p(T x, T y) \leq \max \{p(x, y), p(x, x), p(y, y)\}-\psi(p(x, y), p(x, x)) .
$$

Thus, we have $\psi(p(x, y), p(x, x))=0$. Hence $p(x, y)=p(x, x)=0$. By $\left(p_{2}\right)$, we have $p(y, y)=0$. Therefore by $\left(p_{1}\right)$, we get that $x=y$.

By taking $\psi:[0,+\infty) \times[0,+\infty) \rightarrow[0,+\infty)$ via $\psi(s, t)=(1-r) \max \{s, t\}$ where $r \in[0,1)$ in Theorem 2.1, we have the following result:

Corollary 2.2. Let $(X, p)$ be a complete partial metric space and $T: X \rightarrow X$ be a mapping satisfying

$$
p(T x, T y) \leq r \max \left\{p(x, y), p(x, T x), p(y, T y), \frac{1}{2}[p(x, T y)+p(T x, y)]\right\}
$$

for all $x, y \in X$. If $r \in[0,1)$, then $T$ has a unique fixed point.

As a special case of Corollary (2.2), we have the following result of Matthews.

Corollary 2.3. [22] Let $(X, p)$ be a complete partial metric space and $T: X \rightarrow X$ be a mapping satisfying $p(T x, T y) \leq r p(x, y)$ for all $x, y \in X$. If $r \in[0,1)$, then $T$ has a unique fixed point.

As a direct result of Theorem 2.1, we have the following result.

Corollary 2.4. Let $(X, p)$ be a complete partial metric space and $T: X \rightarrow X$ be a mapping satisfying

$$
\begin{aligned}
p(T x, T y) \leq & \max \left\{p(x, T x), p(y, T y), \frac{1}{2}[p(x, T y)+p(T x, y)]\right\} \\
& -\psi(p(x, y), p(x, T x)), \quad \forall x, y \in X,
\end{aligned}
$$

where $\psi:[0, \infty) \times[0,+\infty) \rightarrow[0, \infty)$ is a continuous mapping such that $\psi(t, s)=0$ if and only if $t=s=0$. Then $T$ has a unique fixed point.

Now, we introduced an example to support the useability of our results. 
Example 2.5. Let $X=[0,+\infty)$. Define the partial metric space on $X$ by $p(x, y)=\max \{x, y\}$. Also, define the mapping $T: X \rightarrow X$ by $T(x)=\frac{x^{2}}{1+x}$ and the function $\psi:[0,+\infty) \times[0,+\infty) \rightarrow[0,+\infty)$ by $\psi(s, t)=\frac{s+t}{2+s+t}$. Then

1. $(X, p)$ is a complete partial metric space.

2. $T$ satisfies 2.15 of Corollary 2.4.

3. If we replace $p$ by $p^{s}$ in 2.15) of Corollary 2.4 then $T$ does not satisfy 2.15) of Corollary 2.4 .

Proof. For (1) see Ref. [1]. To prove (2), suppose $y \leq x$. Then

$$
\begin{aligned}
& p(T x, T y)=\max \left\{\frac{x^{2}}{1+x}, \frac{y^{2}}{1+y}\right\}=\frac{x^{2}}{1+x}, \\
& \max \{p(x, T x), p(y, T y)\}=\max \{x, y\}=x
\end{aligned}
$$

and

$$
\psi(p(x, y), p(x, T x))=\psi(x, x)=\frac{2 x}{2+2 x}
$$

Since

$$
\frac{x^{2}}{1+x} \leq x-\frac{2 x}{2+2 x}=\frac{x^{2}}{1+x},
$$

we have $T$ satisfies 2.15 of Corollary 2.4

To prove (3), notice that

$$
p^{s}(x, y)=2 p(x, y)-p(x, x)-p(y, y)=2 \max \{x, y\}-x-y=|x-y| .
$$

Now, take $x=1$ and $y=0$. Then

$$
\begin{gathered}
p^{s}(T 1, T 0)=p^{s}\left(\frac{1}{2}, 0\right)=\frac{1}{2} \\
\max \left\{p^{s}(1, T 1), p^{s}(0, T 0)\right\}=\max \left\{p^{s}\left(1, \frac{1}{2}\right), p^{s}(0,0)\right\}=\frac{1}{2}
\end{gathered}
$$

and

$$
\psi\left(p^{s}(1,0), p^{s}(1, T 1)\right)=\psi\left(1, \frac{1}{2}\right)=\frac{3}{7} .
$$

Since $\frac{1}{2}$ is not less or equal $\frac{1}{2}-\frac{3}{7}$, we get that (3) does hold for $x=1$ and $y=0$.

\section{Acknowledgements:}

The authors thank the referee for the valuable comments and suggestions.

\section{References}

[1] I. Altun and A. Erduran, Fixed point theorems for monotone mappings on partial metric spaces, Fixed Point Theory Appl. 2011 (2011), Article ID 508730, 10 pages. 2

[2] I. Altun and H. Simsek, Some fixed point theorems on dualistic partial metric spaces, J. Adv. Math. Stud. 1(2008), 1-8

[3] I. Altun, F. Sola and H. Simsek, Generalized contractions on partial metric spaces, Topology Appl. 157(18)(2010), 2778-2785.

[4] H. Aydi, Some fixed point results in ordered partial metric spaces, accepted in J. Nonlinear Sci. Appl.(2011).

[5] H. Aydi, E. Karapinar and W. Shatanawi, Coupled fixed point results for $(\psi, \phi)$-weakly contractive condition in ordered partial metric spaces, Computer Math. Appl, 62 (2011), 4446-4460.

[6] V. Berinde, Some remarks on a fixed point theorem for C iric-type almost contractions, Carpathian J. Math. 25(2009), 157-62. 1 
[7] V. Berinde, General constructive fixed point theorems for Ciric-type almost contractions in metric spaces, Carpathian J. Math. 24 (2008),10-9. 1

[8] LjB. Ćirić, Generalized contractions and fixed-point theorems, Publ. IInst Math. (Beograd) 12 (1971), 19-26. 1

[9] LjB. Ćirić, A generalization of Banachs contraction principle, Proc Amer. Math. Soc. 45 (1974), 267-73.

[10] LjB. Ćirić, On contraction type mappings, Math Balkanica 1 (1971), 527.

[11] LjB. Ćirić, Fixed point theory. Contraction Mapping principle, FME Press, Beograd, 2003.

[12] LjB. Ćirić, Coincidence and fixed points for maps on topological spaces, Topol. Appl. 154 (2007), 3100-3106.

[13] LjB. Ćirić, Fixed point theorems for multi-valued contractions in complete metric spaces, J. Math. Anal. Appl. 348 (2008), 499-507.

[14] LjB. Ćirić, Non-self mappings satisfying nonlinear contractive condition with applications, Nonlinear Anal. 71 (2009), 2927-2935.

[15] LjB. Cirić, M. Abbas, R. Saadati and N. Hussain, Common fixed points of almost generalized contractive mappings in ordered metric spaces, Appl. Math. Comput. 227 (12) (2011), 5784-5789.

[16] LjB. Ćirić, N. Cakić, M. Rajovi and J. S. Ume, Monotone generalized nonlinear contractions in partially ordered metric spaces, Fixed Point Theory Appl. 2008(2008) Article ID 131294, 11 pages. 1

[17] LjB. Ćirić, B. Samet, H. Aydi and C. Vetro, Common fixed points of generalized contractions on partial metric spaces and an application, Appl. Math. Comput. 218 (2011), 2398-2406.

[18] R. Heckmann, Approximation of metric spaces by partial metric spaces, Appl. Categ. Structures 7(1999), 71-83.

[19] D. Ilić, V. Pavlović and V. Rakočević, Some new extensions of Banach's contraction princible to partial metric space, Appl. Math. Lett. (2011), doi:10.1016/j.aml.2011.02.025

[20] E. Karapinar, Weak $\phi$-contraction on partial contraction, J. Comput. Anal. Appl. (in press). 1.4

[21] E. Karapınar, Generalizations of Caristi Kirk's Theorem on Partial metric Spaces, Fixed Point Theory Appl. (in press).

[22] S. G. Matthews, Partial metric topology. In, General Topology and its Applications, Proc. 8th Summer Conf., Queen's College (1992). Annals of the New York Academy of Sciences, 728 (1994), 183-197. 1, 1.1, 1.2, 1.3, 2.3

[23] H. K. Nashine, Fixed point results for mappings satisfying $(\psi, \varphi)$-weakly contractive condition in ordered partial metric spaces, accepted in Math. Slovaca (2011).

[24] O. Valero, On Banach fixed point theorems for partial metric spaces, Applied General Topology 6(2) (2005),229240.

[25] S. Oltra, O. Valero, Banach's fixed point theorem for partial metric spaces, Rendiconti dell'Istituto di Matematica dell'Universit di Trieste 36(1-2)( 2004), 17-26.

[26] S. Romaguera, A Kirk type characterization of completeness for partial metric spaces, Fixed Point Theory Appl. 2010(2010), Article ID 493298, 6 pages.

[27] B. Samet and C. Vetro, An integral version of Ćirić s fixed point theorem, Mediterr J Math. doi:10.1007/s00009011-0120-1. 1

[28] W. Shatanawi, B. Samet and M. Abbas, Coupled fixed point theorems for mixed monotone mappings in ordered partial metric spaces, Math. Comput. Mod. (2011), doi:10.1016/j.mcm.2011.08.042. 